\title{
Modeling of MEMS Resonator Piezoelectric Disc Partially Covered with Electrodes
}

\author{
Ismail Naciri ${ }^{1,}$,, Lahoucine Elmaimouni ${ }^{1}$, Jean-Etienne Lefebvre ${ }^{2}$, \\ Faniry Emilson Ratolojanahary ${ }^{3}$, Mohamed Rguiti ${ }^{4}$, Tadeusz Gryba ${ }^{2}$ \\ ${ }^{1}$ Laboratoire Sciences Ingénierie et Energie, Energie Renouvelable, Microsystèmes Acoustique et Micanique, Polydisciplinary Faculty of \\ Ouarzazate, Ibn Zohr University, Morocco \\ ${ }^{2}$ The Institute of Electronics, Microelectronics and Nanotechnology, Opto-Acousto-Electronic Department, University of Valenciennes, \\ France \\ ${ }^{3}$ Laboratory of Applied Physics, Fianarantsoa University, Madagascar \\ ${ }^{4}$ Laboratoire des Matériaux Céramiques et Procédés Associés, Université de Valenciennes, Maubeuge, France
}

\section{Email address:}

nacirismail@gmail.com (I. Naciri), la_elmaimouni@yahoo.fr (L. Elmaimouni)

${ }^{*}$ Corresponding author

\section{To cite this article:}

Ismail Naciri, Lahoucine Elmaimouni, Jean-Etienne Lefebvre, Mohamed Rguiti, Faniry Emilson Ratolojanahary, Tadeusz Gryba. Modeling of MEMS Resonator Piezoelectric Disc Partially Covered with Electrodes. American Journal of Mechanics and Applications.

Vol. 4, No. 1, 2016, pp. 1-9. doi: 10.11648/j.ajma.20160401.11

Received: August 31, 2016; Accepted: September 26, 2016; Published: October 19, 2016

\begin{abstract}
The Legendre polynomial method has been extended to the modeling of MEMS resonator disc partially covered with electrodes. The disc has been divided into two areas: one with electrodes and the other without electrodes. For each area, The Maxwell equations and the piezoelectric constitutive equations of motion are studied and solved to yield a frequency response and electrical behavior of the MEMS resonator applying a semi analytical method based on a Legendre polynomials series and trigonometric functions. However, the method allows incorporating the boundary conditions directly into the governing equations by assuming position-dependent of elastic constants, mass density and delta functions. The alternating electrical source is described by specific terms which are also introduced into the equation of motion. The formalism has been developed which allows for both harmonic and modal analyses. In order to validate our polynomial approach, numerical results are presented such as resonant and anti-resonant frequencies, electric input admittance, electromechanical coupling coefficient and field profiles of fully and partially metallized PZT5A resonator discs. The results obtained were compared with those obtained by an approximated analytical method. The developed software proves to be very efficient to retrieve the contour modes of all orders.
\end{abstract}

Keywords: MEMS Resonators, Legendre Polynomial Approach, Centralized Metallization, Piezoelectric Resonator Disc, Electrical Admittance, Resonant, Anti-resonant Frequencies

\section{Introduction}

When a piezoelectric material is subjected to a mechanical strain, electrical charges were generated and conversely. This phenomenon was widely exploited in various engineering applications such as Micro-Electro-Mechanical systems (MEMS) technology. MEMS technology has been obtained significant growth in its field of application such as in electro-optic modulators, ultrasonic detectors, accelerometers, transducers, oscillators, electromechanical sensors and actuators [1-6].

Although, the vibration characteristics of piezoelectric materials are extracted from the piezoelectric constitutive equations, linear piezoelectricity and the Maxwell equations [7-8].

Several methods allow calculating vibration characteristics of piezoelectric devices. In 1967, Eer Nisse [9] presented a vibrational method to analyze the vibrational behavior of piezoelectric disks. C.H.Huang and C.C Ma used Electronic speckle pattern interferometry method to study vibration 
characteristics for piezoelectric cylinders [10]. Kharouf et al [11] studied the vibrational axisymmetric characteristics of hollow cylinders and disks made from piezoelectric materials by using the Rayleigh-Ritz method. These methods give accurate results especially for simple geometries. In the case of complicated geometries, alternative methods are needed. The finite elements method was used to analyze vibrational modes of ceramic disk composed of PZT5A with the diameter to thicknesses ratio range from 0.2 to 10 [12]. By the same method, Guo et al [13] have been calculated the vibration characteristics of disks composed of PZT5A with diameter to thicknesses ratio ranging from 10 to 20 .

Moreover, Legendre polynomial method is one of the most methods used recently to check the electrical response of piezoelectric resonators and gives excellent precision for various structures [14-21]. This method is based on expressing the components of electrical and mechanical displacement by double series of Legendre polynomials.

In this article, polynomial method was extended for studying the frequency spectrum of a piezoelectric PZT5A disc resonator divided into two areas. Centralized area is electroded whereas the annular area is free of electrodes.

\section{Formulation of the Problem}

As shown in figure 1, the studied geometrical configuration is a homogeneous cylinder of finite length, divided into two areas named $\mathrm{N}$ and $\mathrm{M}$. $\mathrm{M}$ area contains electrodes from the top and the bottom. $\mathrm{H}$ and $\mathrm{R}$ are the thickness and the radius of the cylinder respectively.

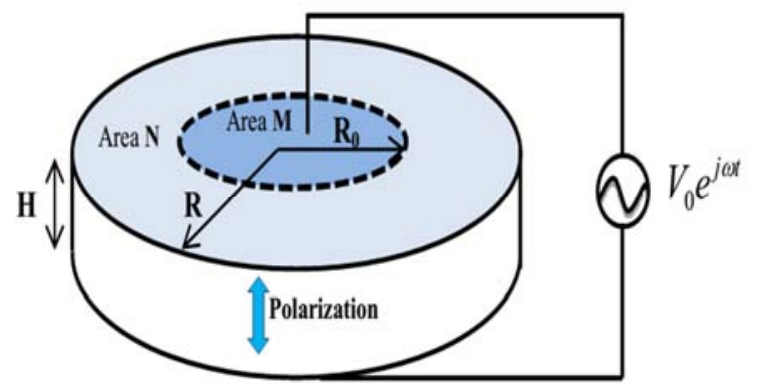

Figure 1. Structure of a piezoelectric disc partially metallized with electric excitation source $V_{0} e^{j \omega t}$.

Studying the structure in cylindrical coordinates $\operatorname{Or} \varphi z$. The z-axis coincides with the axis of anisotropy and the origin is placed at the center of the disc.

The vibration characteristics of a piezoelectric materials are studied by following equations [22-23]:

$$
\begin{aligned}
& \rho \frac{\partial^{2} \vec{U}(M, t)}{\partial t^{2}}=\nabla \cdot \sigma(M) \quad \text { and } \quad \frac{\partial D_{i}}{\partial x_{i}}=0 \\
& \pi\left(q_{3}\right)= \begin{cases}1 & -1 \leq q_{3} \leq 1 \\
0 & \text { Elsewhere }\end{cases}
\end{aligned}
$$

where $D_{i}=\left[\begin{array}{lll}D_{r}, & D_{\varphi}, D_{z}\end{array}\right]$ are the components of electrical displacements and $\sigma$ denotes the stress tensor. $\vec{U}(M, t)=(\vec{u}, \vec{v}, \vec{w})$ are the components of mechanical displacement in the radial, circumferential and axial direction.

Under the assumption of small deformations, the strain mechanical displacements relations in terms of cylindrical coordinates system are expressed by [24].

$$
\begin{aligned}
& S_{r r}=\frac{\partial u}{\partial r} \quad 2 S_{\varphi z}=\frac{\partial v}{\partial z}+\frac{1}{r} \frac{\partial w}{\partial \varphi} \quad 2 S_{r z}=\frac{\partial u}{\partial z}+\frac{\partial w}{\partial r} \\
& S_{\varphi \varphi}=\frac{u}{r}+\frac{1}{r} \frac{\partial v}{\partial \varphi} \quad 2 S_{r \varphi}=\frac{1}{r} \frac{\partial u}{\partial \varphi}+\frac{\partial v}{\partial r}-\frac{v}{r} \quad S_{z z}=\frac{\partial w}{\partial z}
\end{aligned}
$$

Along this paper, we adopt the following change of variables:

$$
\begin{gathered}
\bar{\varepsilon}_{i j}=\varepsilon_{i j} / \varepsilon_{33}, \bar{C}_{i j}=C_{i j} / C_{33}^{D}, \bar{e}_{i j}=e_{i j} / \sqrt{C_{33}^{D} \varepsilon_{33}} \\
\bar{D}_{k}=D_{k} / \varepsilon_{33}, \bar{\sigma}_{i j}=\sigma_{i j} / C_{33}^{D}, C_{33}^{D}=C_{33}+\varepsilon_{33}^{2} / \varepsilon_{33}
\end{gathered}
$$

Normalized mechanical displacement components: $\bar{u}=u(m) \times 10^{10}, \bar{v}=v(m) \times 10^{10}, \bar{w}=w(m) \times 10^{10}$.

$q_{1}=R / r$ and $q_{3}=2 z / H$.

Equations expressed in (1) can be written as:

$$
\begin{gathered}
\rho \frac{\partial^{2} u}{\partial t^{2}}=\frac{\partial \sigma_{r r}}{\partial r}+\frac{\partial \sigma_{r z}}{\partial z}+\frac{1}{r} \frac{\partial \sigma_{r \varphi}}{\partial \varphi}+\frac{\sigma_{r r}-\sigma_{\varphi \varphi}}{r} \\
\rho \frac{\partial^{2} v}{\partial t^{2}}=\frac{\partial \sigma_{r \varphi}}{\partial r}+\frac{\partial \sigma_{\varphi z}}{\partial z}+\frac{1}{r} \frac{\partial \sigma_{\varphi \varphi}}{\partial \varphi}+\frac{2 \sigma_{r \varphi}}{r} \\
\rho \frac{\partial^{2} w}{\partial t^{2}}=\frac{\partial \sigma_{r z}}{\partial r}+\frac{\partial \sigma_{z z}}{\partial z}+\frac{1}{r} \frac{\partial \sigma_{\varphi z}}{\partial \varphi}+\frac{\sigma_{r z}}{r} \\
\frac{1}{r} \frac{\partial\left(r D_{r}\right)}{\partial r}+\frac{1}{r} \frac{\partial\left(D_{\varphi}\right)}{\partial \varphi}+\frac{\partial\left(D_{z}\right)}{\partial z}=0
\end{gathered}
$$

Taking in account the axisymmetric character of electrical source and studied structure, the variation of wave field does not depend on $\varphi$ direction. Moreover, the mechanical displacement in circumferential direction is uncoupled from the electric field. We can only tike in consideration the equations (3a), (3c) and (3d).

Two rectangular window functions $\pi\left(q_{3}\right)$ and $\theta\left(q_{1}\right)$ express the boundary conditions related to our structure.

$$
\theta\left(q_{1}\right)= \begin{cases}1 & 0 \leq q_{1} \leq 1 \\ 0 & \text { Elsewhere }\end{cases}
$$

The equations expressed in (4) are directly incorporated in the constitutive equations of our problem. 
The normalized stress components are expressed by

$$
\begin{gathered}
\bar{\sigma}_{r r}^{(j)}=\left[\bar{C}_{11} \frac{1}{R} \frac{\partial \bar{u}^{(j)}}{\partial q_{1}}+\bar{C}_{12} \frac{1}{R} \frac{\bar{u}^{(j)}}{q_{1}}+\bar{C}_{13} \frac{2}{H} \frac{\partial \bar{w}^{(j)}}{\partial q_{3}}+\bar{e}_{31} \frac{1}{\beta} \frac{2}{H} \frac{\partial \phi^{(j)}}{\partial q_{3}}\right] \cdot 10^{-10} \\
\bar{\sigma}_{\varphi \varphi}^{(j)}=\left[\bar{C}_{12} \frac{1}{R} \frac{\partial \bar{u}^{(j)}}{\partial q_{1}}+\bar{C}_{11} \frac{1}{R} \frac{\bar{u}^{(j)}}{q_{1}}+\bar{C}_{13} \frac{2}{H} \frac{\partial \bar{w}^{(j)}}{\partial q_{3}}+\bar{e}_{31} \frac{1}{\beta} \frac{2}{H} \frac{\partial \phi^{(j)}}{\partial q_{3}}\right] \cdot 10^{-10} \\
\bar{\sigma}_{z z}^{(j)}=\left[\bar{C}_{13} \frac{1}{R} \frac{\partial \bar{u}^{(j)}}{\partial q_{1}}+\bar{C}_{13} \frac{1}{R} \frac{\bar{u}^{(j)}}{q_{1}}+\bar{C}_{33} \frac{2}{H} \frac{\partial \bar{w}^{(j)}}{\partial q_{3}}+\bar{e}_{33} \frac{1}{\beta} \frac{2}{H} \frac{\partial \phi^{(j)}}{\partial q_{3}}\right] \cdot 10^{-10} \\
\bar{\sigma}_{r z}^{(j)}=\left[\bar{C}_{55}\left(\frac{2}{H} \frac{\partial \bar{u}^{(j)}}{\partial q_{3}}+\frac{1}{R} \frac{\partial \bar{w}^{(j)}}{\partial q_{1}}\right)+\bar{e}_{15} \frac{1}{\beta} \frac{1}{R} \frac{\partial \phi^{(j)}}{\partial q_{3}}\right] \cdot 10^{-10}
\end{gathered}
$$

The electric displacement components:

$$
\begin{gathered}
\bar{D}_{q_{1}}^{(j)}=\bar{e}_{15}\left(\frac{2 \beta}{H} \frac{\partial \bar{u}^{(j)}}{\partial q_{3}}\right)+\bar{e}_{15}\left(\frac{\beta}{R} \frac{\partial \bar{w}^{(j)}}{\partial q_{1}}\right)-\bar{\varepsilon}_{11}\left(\frac{1}{R} \frac{\partial \phi^{(j)}}{\partial q_{1}}\right) \\
\bar{D}_{q_{1}}^{(j)}=\bar{e}_{31}\left(\frac{\beta}{R} \frac{\partial \bar{u}^{(j)}}{\partial q_{1}}\right)+\bar{e}_{31}\left(\frac{\beta}{R} \frac{\bar{u}^{(j)}}{q_{1}}\right)+\bar{e}_{33}\left(\frac{2 \beta}{H} \frac{\partial \bar{w}^{(j)}}{\partial q_{3}}\right)-\bar{\varepsilon}_{33}\left(\frac{2}{H} \frac{\partial \phi^{(j)}}{\partial q_{3}}\right)
\end{gathered}
$$

The index $\mathrm{j}=1,2$ refers to area $\mathrm{M}$ and area $\mathrm{N}$ respectively.

Both for the metalized and non-metalized area, we express the field of governing equations (4) and their corresponding boundary, symmetry and continuity conditions.

For the metallized area, we have: $0 \leq q_{1} \leq a_{0}$ and $-1 \leq q_{3} \leq 1$ with $a_{0}=R_{0} / R$

In this area, the boundary, symmetry and continuity conditions are defined by following relations:

$$
\begin{gathered}
\sigma_{r r}^{(1)}\left(q_{1}=a_{0}\right)=\sigma_{r r}^{(2)}\left(q_{1}=a_{0}\right) \\
D_{q_{1}}^{(1)}\left(q_{1}=a_{0}\right)=D_{q_{1}}^{(2)}\left(q_{1}=a_{0}\right) \\
\varphi^{(1)}\left(q_{1}=a_{0}\right)=\varphi^{(2)}\left(q_{1}=a_{0}\right) \\
\sigma_{r z}^{(1)}\left(q_{3}= \pm 1\right)=\sigma_{z z}^{(1)}\left(q_{3}= \pm 1\right)=0 \\
\varphi\left(q_{3}=+1\right)-\varphi\left(q_{3}=-1\right)=V
\end{gathered}
$$

The field of equations (3) becomes:

$$
\begin{aligned}
& \frac{1}{R} \frac{\partial \bar{\sigma}_{r r}^{(1)}}{\partial q_{1}}+\frac{2}{H} \frac{\partial \bar{\sigma}_{r z}{ }^{(1)}}{\partial q_{3}}+\frac{\bar{\sigma}_{r r}^{(1)}-\bar{\sigma}_{\varphi \varphi}^{(1)}}{R q_{1}}+\frac{1}{R}\left(\bar{\sigma}_{r r}{ }^{(2)}-\bar{\sigma}_{r r}{ }^{(1)}\right) \delta\left(q_{1}-a_{0}\right) \\
& +\frac{2}{H} \bar{\sigma}_{r z}^{(1)} \frac{\partial \pi\left(q_{3}\right)}{\partial q_{3}}=10^{-10} \frac{\rho}{C_{33}^{D}} \frac{\partial^{2} \bar{u}^{(1)}}{\partial t^{2}} \\
& \quad \frac{1}{R} \frac{\partial \bar{\sigma}_{r z}^{(1)}}{\partial q_{1}}+\frac{2}{H} \frac{\partial \bar{\sigma}_{z z}^{(1)}}{\partial q_{3}}+\frac{\bar{\sigma}_{r z}{ }^{(1)}}{R q_{1}}+\frac{1}{R}\left(\bar{\sigma}_{r z}^{(2)}-\bar{\sigma}_{r z}{ }^{(1)}\right) \delta\left(q_{1}-a_{0}\right) \\
& +\frac{2}{H} \bar{\sigma}_{z z}^{(1)} \frac{\partial \pi\left(q_{3}\right)}{\partial q_{3}}=10^{-10} \frac{\rho}{C_{33}^{D}} \frac{\partial^{2} \bar{w}^{(1)}}{\partial t^{2}}
\end{aligned}
$$




$$
\begin{aligned}
& \frac{1}{R q_{1}} \frac{\partial}{\partial q_{1}}\left(q_{1} \bar{D}_{q_{1}}{ }^{(1)}\right)+\frac{2}{H} \frac{\partial \bar{D}_{q_{3}}{ }^{(1)}}{\partial q_{3}}+\frac{1}{R}\left(\bar{D}_{q_{1}}{ }^{(2)}-\bar{D}_{q_{1}}{ }^{(1)}\right) \delta\left(q_{1}-a_{0}\right) \\
& +\frac{2}{H} \bar{D}_{q_{3}}{ }^{(1)} \frac{\partial \pi\left(q_{3}\right)}{\partial q_{3}}=0
\end{aligned}
$$

For the non-metalized area, we have: $a_{0} \leq q_{1} \leq 1$ and $-1 \leq q_{3} \leq 1$ with $a_{0}=R_{0} / R$

We express also the boundary, symmetry and continuity conditions defined by following relations:

$$
\begin{gathered}
\sigma_{r z}^{(2)}\left(q_{1}=a_{0}\right)=\sigma_{r z}^{(1)}\left(q_{1}=a_{0}\right) ; \sigma_{r z}^{(2)}\left(q_{3}= \pm 1\right)=0 \\
\sigma_{r r}^{(2)}\left(q_{1}=+1\right)=\sigma_{z z}^{(2)}\left(q_{3}= \pm 1\right)=\sigma_{r z}^{(2)}\left(q_{1}= \pm 1\right)=0 \\
D_{q_{1}}^{(2)}\left(q_{1}=a_{0}\right)=D_{q_{1}}^{(1)}\left(q_{1}=a_{0}\right) ; D_{q_{3}}^{(2)}\left(q_{3}= \pm 1\right)=D_{q_{1}}^{(2)}\left(q_{1}=1\right)=0
\end{gathered}
$$

The field of equations (3) becomes:

$$
\begin{aligned}
& \frac{1}{R} \frac{\partial \bar{\sigma}_{r r}^{(2)}}{\partial q_{1}}+\frac{2}{H} \frac{\partial \bar{\sigma}_{r z}{ }^{(2)}}{\partial q_{3}}+\frac{\bar{\sigma}_{r r}{ }^{(2)}-\bar{\sigma}_{\varphi \varphi}{ }^{(2)}}{R q_{1}}+\frac{1}{R}\left(\bar{\sigma}_{r r}^{(2)}-\bar{\sigma}_{r r}{ }^{(1)}\right) \delta\left(q_{1}-a_{0}\right) \\
& +\frac{2}{H} \bar{\sigma}_{r z}{ }^{(2)} \frac{\partial \pi\left(q_{3}\right)}{\partial q_{3}}-\frac{1}{R} \bar{\sigma}_{r r}^{(2)} \delta\left(q_{1}-1\right)=10^{-10} \frac{\rho}{C_{33}^{D}} \frac{\partial^{2} \bar{u}^{(2)}}{\partial t^{2}} \\
& \frac{1}{R} \frac{\partial \bar{\sigma}_{r z}{ }^{(2)}}{\partial q_{1}}+\frac{2}{H} \frac{\partial \bar{\sigma}_{z z}^{(2)}}{\partial q_{3}}+\frac{\bar{\sigma}_{r z}{ }^{(2)}}{R q_{1}}+\frac{1}{R}\left(\bar{\sigma}_{r z}^{(2)}-\bar{\sigma}_{r z}{ }^{(1)}\right) \delta\left(q_{1}-a_{0}\right) \\
& +\frac{2}{H} \bar{\sigma}_{z z}^{(2)} \frac{\partial \pi\left(q_{3}\right)}{\partial q_{3}}-\frac{1}{R} \bar{\sigma}_{r z}^{(2)} \delta\left(q_{1}-1\right)=10^{-10} \frac{\rho}{C_{33}^{D}} \frac{\partial^{2} \bar{w}^{(2)}}{\partial t^{2}} \\
& \frac{1}{R q_{1}} \frac{\partial}{\partial q_{1}}\left(q_{1} \bar{D}_{q_{1}}{ }^{(2)}\right)+\frac{2}{H} \frac{\partial \bar{D}_{q_{3}}{ }^{(2)}}{\partial q_{3}}+\frac{1}{R}\left(\bar{D}_{q_{1}}{ }^{(2)}-\bar{D}_{q_{1}}^{(1)}\right) \delta\left(q_{1}-a_{0}\right) \\
& +\frac{2}{H} \bar{D}_{q_{3}}{ }^{(2)} \frac{\partial \pi\left(q_{3}\right)}{\partial q_{3}}-\frac{1}{R} \bar{D}_{q_{1}}^{(2)} \delta\left(q_{1}-1\right)=0
\end{aligned}
$$

Mechanical and electrical displacement components for area $\mathrm{M}$ and $\mathrm{N}$ are defined respectively as follow:

$$
\begin{aligned}
& \left\{\begin{aligned}
\bar{u}^{(M)}\left(q_{1}, q_{3}, t\right) & =\sum_{m n} p_{m, 2 n}^{\left(q_{1}, 1\right)} Q_{m}^{(1)}\left(q_{1}\right) Q_{2 n}\left(q_{3}\right) e^{j \omega t} \\
\bar{w}^{(M)}\left(q_{1}, q_{3}, t\right) & =\sum_{m n} p_{m, 2 n+1}^{\left(q_{3}, 1\right)} Q_{m}^{(1)}\left(q_{1}\right) Q_{2 n+1}\left(q_{3}\right) e^{j \omega t} \\
\phi^{(M)}\left(q_{1}, q_{3}, t\right) & =\left[\frac{V_{0}}{2} q_{3}^{3}+\left(q_{3}^{2}-1\right) \sum_{m n} r_{m, 2 n+1}^{(1)} Q_{m}^{(1)}\left(q_{1}\right) Q_{2 n+1}\left(q_{3}\right)\right] e^{j \omega t}
\end{aligned}\right. \\
& \left\{\begin{array}{l}
\bar{u}^{(N)}\left(q_{1}, q_{3}, t\right)=\bar{u}^{(M)}\left(a_{0}, q_{3}, t\right)+\left(q_{1}-a_{0}\right) \sum_{m n} p_{m, 2 n}^{\left(q_{1}, 2\right)} Q_{m}^{(2)}\left(q_{1}\right) Q_{2 n}\left(q_{3}\right) e^{j \omega t} \\
\bar{w}^{(N)}\left(q_{1}, q_{3}, t\right)=\bar{w}^{(M)}\left(a_{0}, q_{3}, t\right)+\left(q_{1}-a_{0}\right) \sum_{m n} p_{m, 2 n+1}^{\left(q_{3}, 2\right)} Q_{m}^{(2)}\left(q_{1}\right) Q_{2 n+1}\left(q_{3}\right) e^{j \omega t} \\
\phi^{(N)}\left(q_{1}, q_{3}, t\right)=\phi^{(M)}\left(a_{0}, q_{3}, t\right)+\left(q_{1}-a_{0}\right) \sum_{m n} r_{m, 2 n+1}^{(2)} Q_{m}^{(2)}\left(q_{1}\right) Q_{2 n+1}\left(q_{3}\right) e^{j \omega t}
\end{array}\right.
\end{aligned}
$$

where: 


$$
\begin{gathered}
Q_{m}^{(1)}\left(q_{m}\right)=\sqrt{(2 m+1) / a_{0}} \times P_{m}\left(\left(2 q_{1} / a_{0}\right)-1\right) Q_{m}^{(2)}\left(q_{3}\right)=\sqrt{(2 m+1) /\left(1-a_{0}\right)} P_{m}\left(2 q_{1} /\left(1-a_{0}\right)-\left[\left(1+a_{0}\right) /\left(1-a_{0}\right)\right]\right) \\
Q_{m}\left(q_{3}\right)=[(2 m+1) / 2] P_{m}\left(q_{3}\right) \text { and } Q_{n}\left(q_{3}\right)=[(2 m+1) / 2] P_{n}\left(q_{3}\right)
\end{gathered}
$$

are the Legendre polynomials of degree $\mathrm{m}$ and $\mathrm{n}$ respectively. The expansions coefficients $p_{m, 2 n}^{\left(q_{i}, i\right)}$ and $r_{m, n}^{(i)}$ are in Angstrom and Volts, respectively. The term $\left(q_{3}^{2}-1\right)$ in the equation of electric potential ensure that $\phi^{(1)}\left(q_{1}, q_{3}=1, t\right)-\phi^{(1)}\left(q_{1}, q_{3}=-1, t\right)=V$. The delta functions $\delta\left(q_{1}=a_{0}\right), \quad \delta\left(q_{1}=1\right)$ and $\delta\left(q_{3}= \pm 1\right)$ refers to the boundary and continuity conditions in the studied piezoelectric disc. Those functions are multiplied by different components of the electrical displacement and mechanical stress components.

Substituting the mechanical and electrical displacements (8) and (9) in the field of equations (6) and (7) respectively for area $\mathrm{M}$ and area $\mathrm{N}$ and multiplying all equations by $Q_{j}^{(*, 1)}\left(q_{1}\right) \times Q_{k}^{*}\left(q_{3}\right) \cdot \mathrm{j}$ and $\mathrm{k}$ runs from 0 to $\mathrm{M}$ and $\mathrm{N}$ respectively. Governing equations (6) and (7) are integrated over $\left(q_{1}\right)$ from 0 to $a_{0}$ for metalized area, from $a_{0}$ to 1 for non-metalized area while $\left(q_{3}\right)$ runs from -1 to 1 . This formulation allows obtaining a system of $6 \times(M+1) \times(N+1) \quad$ equations with $6 \times(M+1) \times(N+1)$ unknowns. Obtained system can be rewrite as following form:

$$
\begin{gathered}
I_{m n j k} P_{m, n}^{(b)}+J_{m n j k} r_{m, n}+f r_{j k} V_{0}=-\pi^{2} \Omega^{2} M_{m n j k} P_{m, n}^{(b)} \\
G_{m n j k} p_{m, n}^{(b)}+K_{m n j k} r_{m, n}+f_{j k}^{2,5} V_{0}=0
\end{gathered}
$$

where:

$$
f r_{j k}=\left[\begin{array}{c}
f 0_{j k} \\
f 1_{j k} \\
f 3_{j k} \\
f 4_{j k}
\end{array}\right] ; f_{j k}^{2,5}=\left[\begin{array}{c}
f 2_{j k} \\
f 5_{j k}
\end{array}\right] ; p_{m, n}^{(b)}=\left[\begin{array}{l}
p_{m, 2 n}^{\left(q_{1}, 1\right)} \\
p_{m, 2 n+1}^{\left(q_{3}, 1\right)} \\
p_{m, 2 n}^{\left(q_{1}, 2\right)} \\
p_{m, 2 n+1}^{\left(q_{3}, 2\right)}
\end{array}\right]
$$

$\Omega$ Denotes the normalized frequency expressed as follow: $\Omega=\omega\left(\frac{H}{\pi}\right) \sqrt{\frac{\rho}{C_{33}^{D}}}$.

Based on foregoing formulation, we present simultaneously modal and harmonic analyses.

\subsection{Harmonic Analyses}

The harmonic analysis allows determining the normalized electric impedance which is expressed by using the expression of displacement current density in our piezoelectric structure $J=i w D_{z}$.

From the average electrical current that flows through the electrode of surface $\mathrm{S}$, we determine the normalized electric input admittance by following relation:

$$
\bar{Y}=\frac{Y}{j \omega C_{0}^{R_{0}}}
$$

Where $C_{0}^{R_{0}}=\frac{\pi R_{0}^{2}}{H} \varepsilon_{33}$ denote the static capacitance of the disc resonator.

From equation 12, we obtain:

$$
\bar{Y}(\Omega)=\frac{I_{0}}{V_{0}}=1-\frac{1}{V_{0}} \alpha_{m, n}^{(b)} p_{m, n}^{b}(\Omega)
$$

The matrix element factors $\alpha_{m, n}^{(b)}$ and $p_{m, n}^{b}(\Omega)$ can be expressed as:

$$
\begin{gathered}
\alpha_{m, n}^{(b)}=\left[\begin{array}{llll}
P 1_{m n} & P 2_{m n} & P 0_{m n} & P 0_{m n}
\end{array}\right] \\
p_{m, n}^{b}(\Omega)=\left[\begin{array}{llll}
p_{m, 2 n}^{\left(q_{1}, 1\right)} & p_{m, 2 n+1}^{\left(q_{3}, 1\right)} & p_{m, 2 n}^{\left(q_{1}, 2\right)} & p_{m, 2 n+1}^{\left(q_{3}, 2\right)}
\end{array}\right]^{t}
\end{gathered}
$$

\subsection{Modal Analyses}

Through modal analyses, resonant $\left(\Omega_{r}\right)$ and anti-resonant frequencies $\left(\Omega_{a}\right)$ are calculated by following relations, respectively.

$$
\begin{gathered}
\Omega_{r}^{2} p_{m, n}^{(b)}=\frac{1}{\pi^{2}} I I_{m n j k}\left[M_{m n j k}\right]^{-1} \\
{\left[\Omega_{a}^{2}\right]=\frac{1}{\pi^{2}}\left[M_{m n j k}\right]^{-1}\left(J J_{m n j k} \alpha_{m, n}^{(b)}\left(\Omega_{A n t}\right)-I I_{m n j k}\right)}
\end{gathered}
$$

where $I I_{m n j k}=I_{m n j k}-J_{m n j k}\left[K_{m n j k}\right]^{-1} G_{m n j k}$ and $J J_{m n j k}=J_{m n j k}\left[K_{m n j k}\right]^{-1} f_{j k}^{2,5}-f r_{j k}$

\section{Numerical Results}

We start our calculations with properties in table (1), the computer program is established using Matlab software.

Table 1. Physical properties of PZT5A.

\begin{tabular}{ll}
\hline Parameter & PZT5A \\
\hline$\rho\left(10^{+3} \mathrm{~kg} \cdot \mathrm{m}^{-3}\right)$ & 7.750 \\
Stiffness constant $C_{i j}\left(\times 10^{11} \mathrm{~N} / \mathrm{m}^{2}\right)$ & \\
$\mathrm{C}_{11}$ & 1.21 \\
$\mathrm{C}_{12}$ & 0.754 \\
$\mathrm{C}_{13}$ & 0.752 \\
$\mathrm{C}_{33}$ & 1.11 \\
$\mathrm{C}_{44}$ & 0.211 \\
$\mathrm{C}_{66}=\left(\mathrm{C}_{11}-\mathrm{C}_{12}\right) / 2$ & 0.226 \\
${\text { Piezoelectric constant } e_{i j}\left(C . \mathrm{m}^{-2}\right)}$ \\
\hline
\end{tabular}




\begin{tabular}{ll}
\hline Parameter & PZT5A \\
\hline $\mathrm{e}_{15}$ & 12.3 \\
$\mathrm{e}_{31}$ & -5.4 \\
$\mathrm{e}_{33}$ & 15.8 \\
Permittivity $\varepsilon_{i j}\left(\times 10^{-11} F . m^{-1}\right)$ & \\
$\varepsilon_{11}$ & 811.026 \\
$\varepsilon_{33}$ & 734.88 \\
\hline
\end{tabular}

The radius and the thickness of the studies resonator disc are $\mathrm{H}=1 \mathrm{~mm}, \mathrm{R}=15 \mathrm{~mm}$. The truncation number for our calculation is limited on $\mathrm{M}=\mathrm{N}=12$.

\subsection{Resonant and Antiresonant Frequencies}

The obtained results using polynomial approach are compared with results calculated analytically [25]. Analytical method is presented in appendix A. Tables 2 and 3 show resonant $\left(f_{r}\right)$ and anti-resonant frequencies $\left(f_{a}\right)$ for the first five contour modes of a piezoelectric disc partially metallized with a metallization rate $80 \%$. Associated accuracy is calculated by following relations:

$$
\begin{gathered}
\varepsilon_{r}(\%)=\left|\left(f_{r_{-} \text {analy }}-f_{r_{-} \text {poly }}\right) / f_{r_{-} \text {analy }}\right| \times 100 \\
\varepsilon_{a}(\%)=\left|\left(f_{a_{-} \text {analy }}-f_{a_{-} \text {poly }}\right) / f_{a_{-} \text {analyl }}\right| \times 100
\end{gathered}
$$

Table 4 reveals the electromechanical coupling coefficient of the first five contour modes. This coefficient is calculated for a specific pairs of $f_{r}, f_{a}$ by: $k^{2}=\sqrt{\left(f_{a}^{2}-f_{r}^{2}\right) / f_{a}}$.

Table 2. Normalized resonant frequencies of the first five contour modes, $M=N=15$.

\begin{tabular}{lccc}
\hline Mode & $\boldsymbol{f}_{\boldsymbol{r}_{-} \text {analy }}$ & $\boldsymbol{f}_{\boldsymbol{r}_{-} \boldsymbol{p o l y}}$ & $\boldsymbol{\varepsilon}_{\boldsymbol{r}} \%$ \\
\hline 1 & 66.99604 & 67.00521 & 0.0137 \\
2 & 174.7677 & 174.8456 & 0.0446 \\
3 & 278.6883 & 278.7598 & 0.0257 \\
4 & 376.8626 & 377.0021 & 0.0370 \\
5 & 475.0369 & 475.1246 & 0.0185 \\
\hline
\end{tabular}

Table 3. Normalized anti-resonant frequencies of the first five contour modes, $M=N=15$.

\begin{tabular}{lccc}
\hline Mode & $\boldsymbol{f}_{\boldsymbol{a}_{-} \text {analy }}$ & $\boldsymbol{f}_{\boldsymbol{a}_{-} \text {poly }}$ & $\boldsymbol{\varepsilon}_{\boldsymbol{a}} \%$ \\
\hline 1 & 80.36358 & 81.00870 & 0.8028 \\
2 & 176.6346 & 176.7509 & 0.0658 \\
3 & 269.1923 & 270.0361 & 0.3135 \\
4 & 370.5319 & 370.6517 & 0.0323 \\
5 & 468.7062 & 468.8051 & 0.0211 \\
\hline
\end{tabular}

Table 4. Electromechanical coupling coefficient of the first five contour mode, $M=N=15$.

\begin{tabular}{lll}
\hline Mode & $\boldsymbol{k}_{\text {analy }}^{2}$ & $\boldsymbol{k}_{\text {poly }}^{2}$ \\
\hline 1 & 0.5523 & 0.5620 \\
2 & 0.145 & 0.1464 \\
3 & 0.2679 & 0.2562 \\
4 & 0.1856 & 0.1859 \\
5 & 0.1649 & 0.1647 \\
\hline
\end{tabular}

As shown in those tables, the higher error is less than $0.8 \%$ which means good agreement was found.

\subsection{Normalized Electric Admittance}

Our model allows also determining normalized electric input admittance of piezoelectric disc resonator for different rates of metallization. Figure. 2 and Figure 3 displays normalized electric input admittance of a MEMS PZT5A resonator as a function of normalized frequency of contour modes for metallization rate $50 \%$ and $60 \%$ respectively.

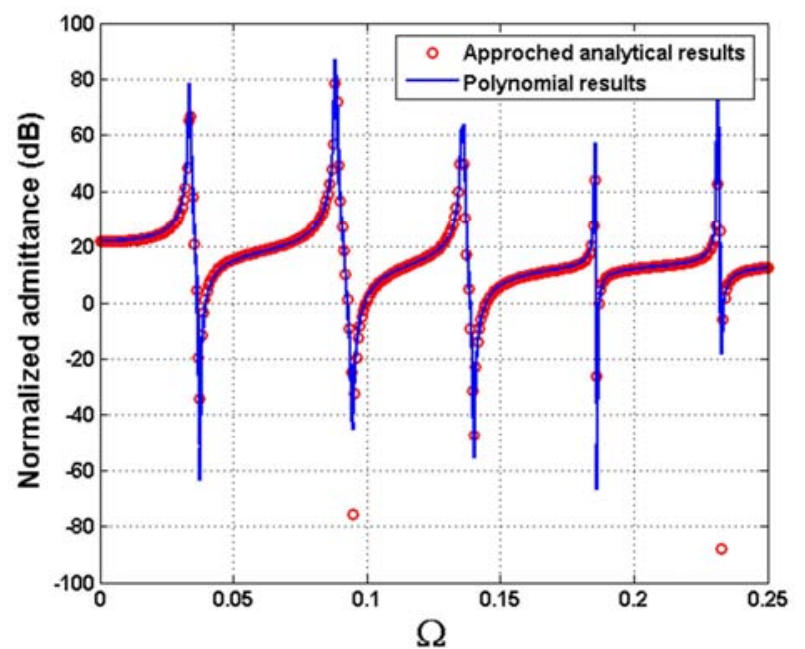

Figure 2. Normalized electric input admittance of PZT5A resonator as a function of normalized frequency of contour modes with. metallization rate of $50 \%, M=N=15$.

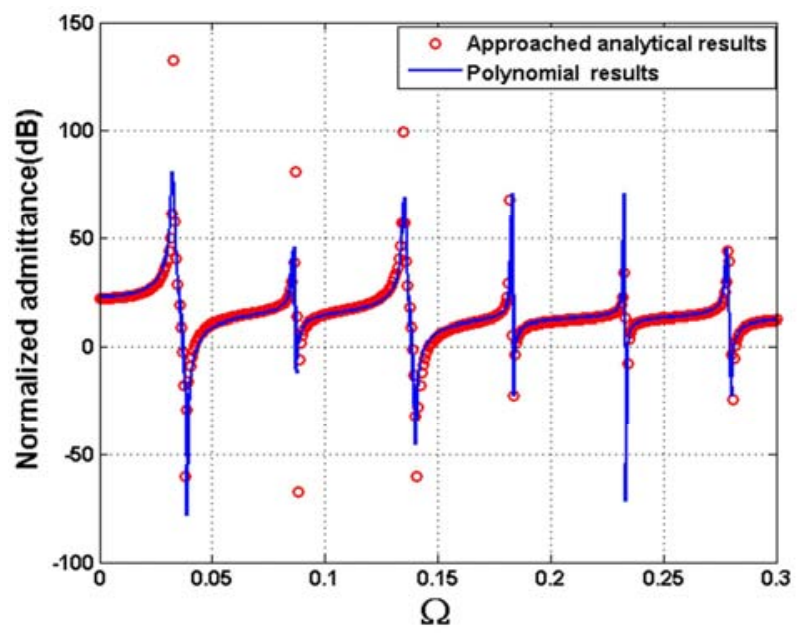

Figure 3. Normalized electric input admittance of PZT5A resonator as a function of normalized frequency of contour modes with metallization rate of $60 \%, M=N=15$.

\subsection{Mechanical and Elctrical Displacements Profiles}

For two values of resonant frequencies $f_{r}=174.8456 \mathrm{kHz}$ and $f_{r}=377.0021 \mathrm{kHz}$, electrical and mechanical displacement profiles are calculated and presented in a 3D configuration for $80 \%$ rate of metallization. Figure 4 and Figure 5 shows the displacements profiles in radial and axial direction respectively. In Figure 6, electrical displacement profiles were presented. 


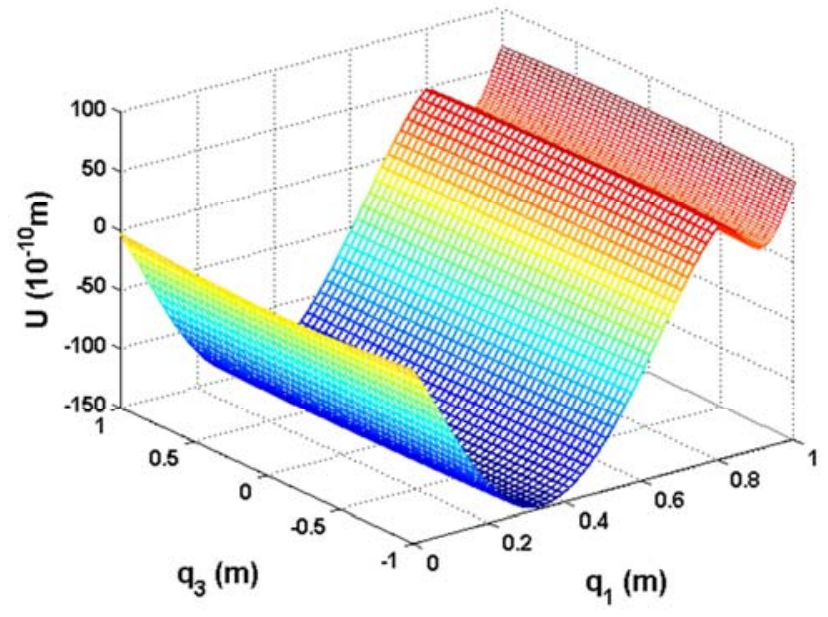

$f_{r}=174.8456 \mathrm{kHz}$

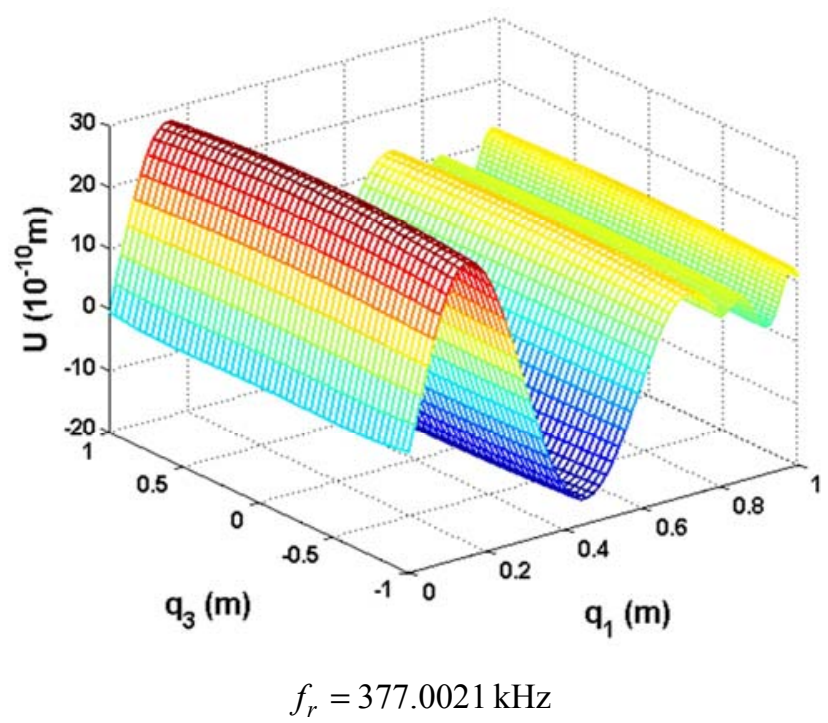

Figure 4. Radial components of displacement profiles for $f_{r}=174.8456 \mathrm{kHz}$ and $f_{r}=377.0021 \mathrm{kHz}, M=N=15$.

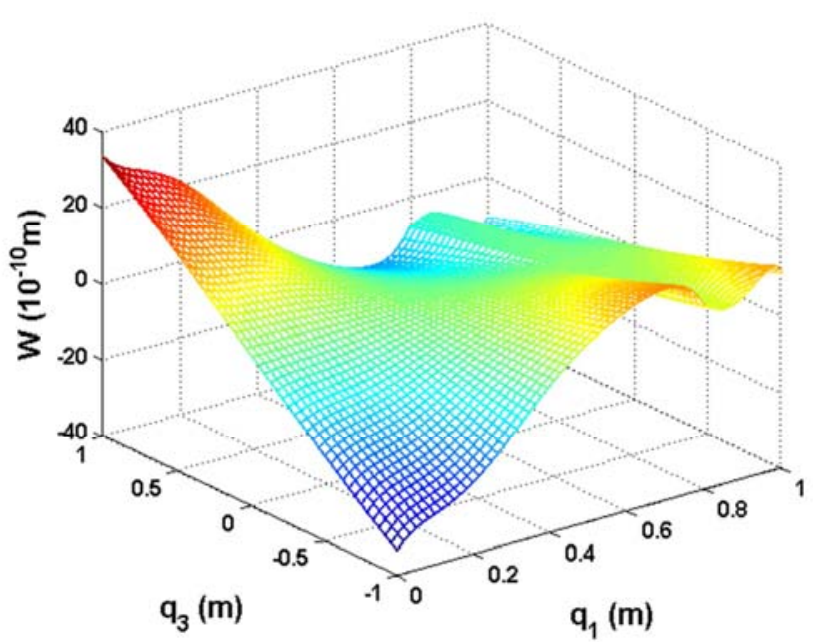

$f_{r}=174.8456 \mathrm{kHz}$

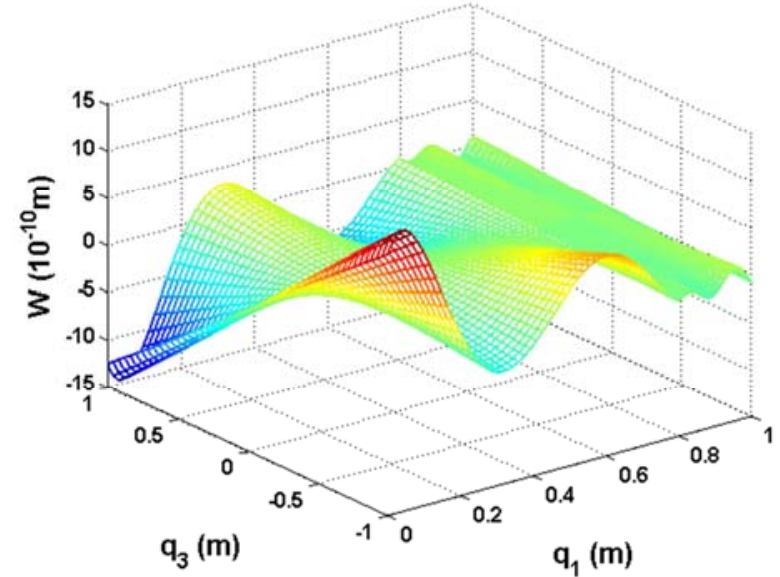

$$
f_{r}=377.0021 \mathrm{kHz}
$$

Figure 5. Axial components of displacement profiles for $f_{r}=174.8456$ $\mathrm{kHz}$ and $f_{r}=377.0021 \mathrm{kHz}, M=N=15$.

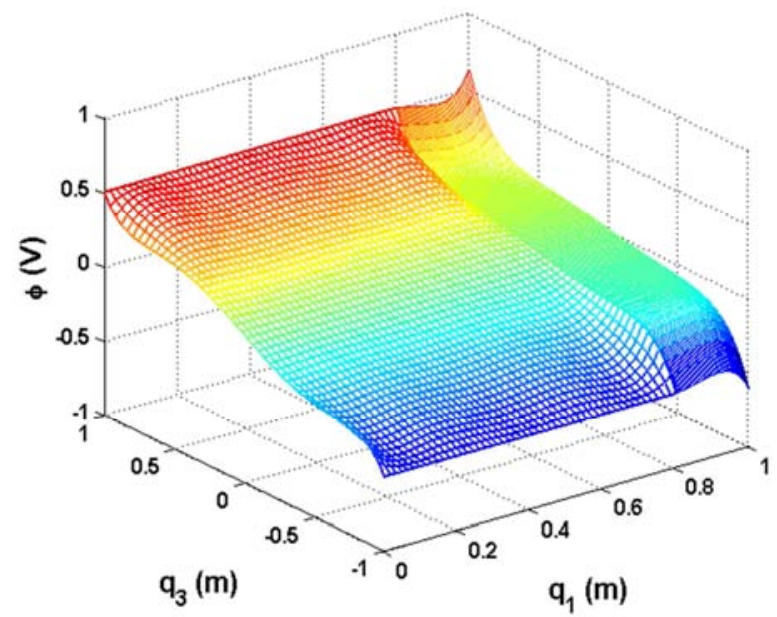

$$
f_{r}=174.8456 \mathrm{kHz}
$$

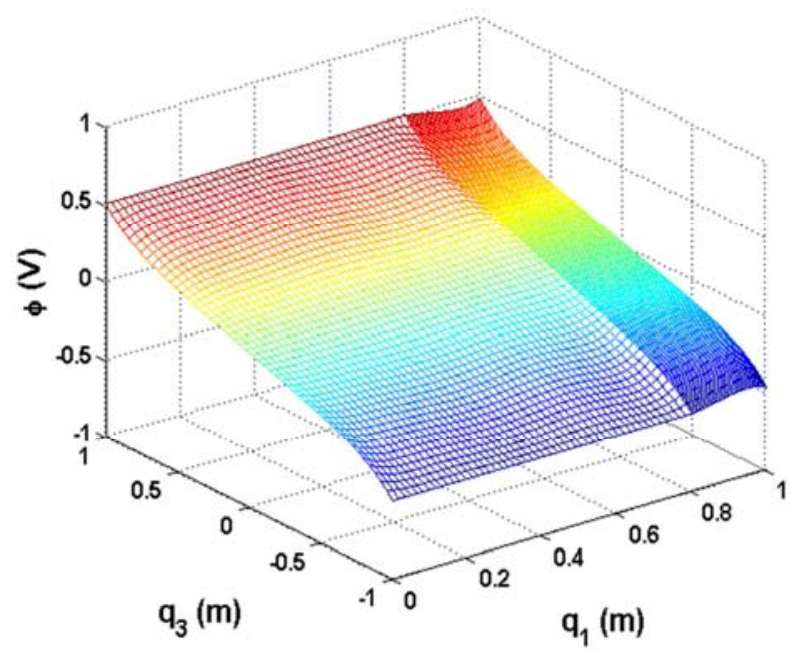

$$
f_{r}=377.0021 \mathrm{kHz}
$$

Figure 6. Electrical displacement profiles into PZT5A resonator for: $f_{r}=174.8456 \mathrm{kHz}$ and $f_{r}=377.0021 \mathrm{kHz}, M=N=15$. 
We can denote that the rate of metallization is an important factor in controlling the frequency variations of the resonator. Therefore, resonant and anti-resonant frequencies increase with the decrease of the length on the electrode.

\section{Conclusion}

Along this paper, Legendre polynomial method was successfully extended for studying the vibrational characteristics of a piezoelectric MEMS resonator partially metallized with centralized metallization. As an optimization tool, Legendre polynomial approach provides satisfied results such as resonant and anti-resonant frequencies, normalized electric impedance, electromechanical coupling coefficient, mechanical and electrical displacement profiles in three dimensional configurations. The formulation is easy to implement in which boundary conditions and electric excitation source are directly incorporated in the equation of motion.

\section{Appendix}

The analytical method used for calculating normalized admittance is developed for a thin disc $(\mathrm{H}<<\mathrm{R})$ made from piezoelectrique material.

$$
\bar{Y}=1+\frac{\left(\frac{d_{31}}{s_{11}^{E}\left(1-v^{E}\right)}\right)^{2}}{\varepsilon_{33}^{T}+2 g_{31} d_{31}} J_{1}\left(\alpha R_{0}\right) \operatorname{Minv}(1,3)
$$

where:

$$
M=\left(\begin{array}{ccc}
M_{11} & M_{12} & M_{13} \\
0 & M_{22} & M_{23} \\
M_{31} & M_{32} & M_{33}
\end{array}\right) \text { where the matrix elements are }
$$

expressed as follow:

$$
\begin{gathered}
M_{11}=J_{1}\left(\alpha R_{0}\right) ; M_{12}=-J_{1}\left(\eta R_{0}\right) ; M_{13}=-Y_{1}\left(\eta R_{0}\right) \\
M_{22}=B_{0} \cdot \eta R . J_{0}(\eta R)-\frac{J_{1}(\eta R)}{s_{11}^{E}\left(1+v^{E}\right)} \\
M_{23}=B_{0} \cdot \eta R \cdot Y_{0}(\eta R)-\frac{Y_{1}(\eta R)}{s_{11}^{E}\left(1+v^{E}\right)} \\
M_{31}=\frac{\alpha R_{0} . J_{1}^{\prime}\left(\alpha R_{0}\right)+v^{E} J_{1}\left(\eta R_{0}\right)}{s_{11}^{E}\left(1+v^{E}\right)^{2}} \\
M_{32}=-\left(\begin{array}{c}
B_{0} \cdot \eta R_{0} . J_{0}\left(\eta R_{0}\right)-\frac{J_{1}\left(\eta R_{0}\right)}{s_{11}^{E}\left(1+v^{E}\right)}
\end{array}\right)
\end{gathered}
$$

$$
M_{33}=-\left(B_{0} \cdot \eta R_{0} \cdot Y_{0}\left(\eta R_{0}\right)-\frac{Y_{1}\left(\eta R_{0}\right)}{s_{11}^{E}\left(1+v^{E}\right)}\right)
$$

Where:

$$
\begin{gathered}
B_{0}=\frac{2-\left(1-v^{E}\right) k_{p}^{2}}{2 s_{11}^{E}\left(1-v^{E}\right)^{2}\left(1-k_{p}^{2}\right)} ; \alpha R_{0}=\omega R_{0} \sqrt{\rho s_{11}^{E}\left(1-v^{E}\right)^{2}} \\
g_{31}=-\frac{d_{31}}{s_{11}^{E}\left(1-v^{E}\right)} ; k_{p}^{2}=\frac{2 d_{31}^{2}}{\left(1-v^{E}\right) s_{11}^{E} \varepsilon_{11}^{T}} ; v^{E}=-\frac{s_{12}^{E}}{s_{11}^{E}}
\end{gathered}
$$

where $J_{0}$ and $J_{1}$ are the Bessel functions of the first kind, $s_{11}^{E}$ and $s_{12}^{E}$ are the elastic compliance constants at constant electrical field. $g_{31}$ and $d_{31}$ are the piezoelectric constants, $v^{E}$ is the Poisson ratio and $\varepsilon_{33}^{T}$ is the dielectric constant at constant stress.

\section{References}

[1] M. Lutz, A. Partridge, P. Gupta, N. Buchan, E. Klaassen, J. McDonald, K. Petersen. "MEMS oscillators for high volume commercial applications", Int. S. State Sen Actuat. Microsyst. (14th Conf. Transducers), pp. 49-52, 2007.

[2] M. H. Tsai, Y. C. Liu, C.M. Sun, C. Wang, C. W. Cheng, W. Fang, "3-Axis CMOS-MEMS accelerometer with vertically integrated fully-differential sensing electrodes", 16th Int. Solid-State S. Actu. Micr. Conf. (TRANSDUCERS), pp. 811814, 2011.

[3] M. Rinaldi, C. Zuniga, G. Piazza, "Ss-DNA functionalized array of AIN contourmode NEMS resonant sensors with single CMOS multiplexed oscillator for subppb detection of volatile organic chemicals", IEEE 24th Int. Conf. MEMS, , pp. 976-979, 2011

[4] T. W. Secord, H. H. Asada, A variable stiffness PZT actuator having tunable resonant frequencies, IEEE Trans. Robot. Vol.26 (6), pp. 993-1005, 2010.

[5] D. W. Greve, J. J. Neumann, I. J. Oppenheim, S. P. Pessiki, D. Ozevin, Robust capacitive MEMS ultrasonics transducers for liquid immersion, IEEE Symp. Ultrason. Vol.1, pp. 581-584, 2003.

[6] D. K. Agrawal, P. Thiruvenkatanathan, J. Yan, A. A. Seshia, Electrically coupled MEMS oscillators, in: Joint Conf. IEEE Int. Freq. Control Eur. Freq. Time Forum (FCS), pp. 1-5, 2011.

[7] H. F. Tiersten, Linear piezoelectric plate vibration, Plenum, New York, 1969.

[8] IEEE Standard on Piezoelectricity, ANSI-IEEE Std. 176, IEEE New York, 1987.

[9] E. P Eer Nisse, "Variational Method for Electroelastic Vibration Analysis," IEEE Trans. Sonics. Ultra. Vol. 14 (4), pp. 153-160, 1967. 
[10] C. H. Huang and C. C. Ma, "Vibration Characteristics for Piezoelectric Cylinders Using Amplitude - Fluctuation Electronic Speckle Pattern Interferometry" AIAA JL Vol. 36 (12), 1998.

[11] Kharouf, N, and Heyliger, P. R., "Axisymmetric Free Vibration of Homogeneous and Laminated Piezoelectric Cylinders," J. Sound. Vib., Vol. 174 (4), pp. 539-561, 1994.

[12] H. A Kunkel, S. Locke, and B. Pikeroen, "Finite-Element Analysis of Vibrational Modes in Piezoelectric Ceramics Disks," IEEE. Trans. Ultrason. Ferr. Freq. Cont, Vol. 37 (4), pp. 316-328, 1990.

[13] N. Guo, P. Cawley and D. Hitchings, "The Finite Element Analysis of the Vibration Characteristics of Piezoelectric Disks,” J Sound. Vib., Vol. 159 (1), pp. 115-138, 1992.

[14] L. Elmaimouni, J. E Lefebvre, V. Zhang, and T. Gryba, "Guided waves in radially graded cylinders: a polynomial approach”, NDT \& E Int, Vol. 38, pp.344-353, 2005.

[15] J. E. Lefebvre, V. Zhang, J. Gazalet, T. Gryba and V. Sadaune, "Acoustic Waves Propagation in Continuous Functionally Graded Plates: An Extension of the Legendre Polynomial Approach" IEEE. Trans. Ultrason. Ferr. Freq. Cont, Vol. 48 (5), pp. 1332-1340, 2001.

[16] J. Yu, J. E. Lefebvre and L. Elmaimouni, Toroidal wave in multilayered spherical curved plates, Journal of Sound and Vibration, Vol. 332 (11), pp. 2816-2830, 2013.

[17] L. Elmaimouni, J. E. Lefebvre, F. E. Ratolojanahary, A. Raherison, T. Gryba and J. Carlier, "Modal analysis and harmonic response of resonators: an extension of a mapped orthogonal functions technique" Wave Motion, Vol. 48 (1), pp. 93-104, 2011.
[18] L. Elmaimouni, J. E. Lefebvre, F. E. Ratolojanahary, A. Raherison, T. Gryba and J. Carlier, "Modal analysis and harmonic response of resonators: an extension of a mapped orthogonal functions technique" Wave Motion, Vol. 48 (1), pp. 93-104 (2011).

[19] L. Elmaimouni, J. E. Lefebvre, F. E. Ratolojanahary A. Raherison, B. Bahani and T. Gryba, "Polynomial approach modeling of resonator piezoelectric disc" Key Engineering Materials, Dynamics of the structures and Non Destructive testing, Vol. 1294 (482), pp. 11-20, 2011.

[20] P. M. Rabotovao, F. E. Ratolojanahary, J. E. Lefebvre, A. Raherison, L. Elmaimouni, T. Gryba, and J. G. Yu, "Modeling of high contrast partially electroded resonators by means of a polynomial approach", J. Applied Physics, Vol. 114 (12), pp. $124502,2013$.

[21] L. Elmaimouni, F. E. Ratolojanahary, J. E. Lefebvre, J. G. Yu, A. Raherison and T. Gryba, "Modeling of MEMS resonator piezoelectric disc by means of an equicharge current source method", Ultra, Vol. 53 (7), pp. 1270-1279, 2013.

[22] B. A Auld, "Acoustic Fields and Waves in Solids" Krieger Publishing Company, Malabar, Florida, 1990.

[23] D. Royer et E Dieulesaint, Ondes élastiques dans les solides, Masson, Paris 1994.

[24] J. Yu, J. E. Lefebvre, Y. Guo, and L. Elmaimouni "Wave Propagartion in the Circumferential Direction of General Multilayered Piezoelectric Cylindrical Plates”. IEEE. Trans. Ultrason. Ferr. Freq. Cont, Vol. 59, pp. 0885-3010, 2012.

[25] N. Guo, "The vibration characteristics of piezoelectric discs", Dissertation, Department of Mechanical Engineering, Imperial College of Science, Technology and Medicine, London, 1989. 